PROCEEDINGS OF THE

AMERICAN MATHEMATICAL SOCIETY

Volume 126, Number 1, January 1998, Pages 25-34

S 0002-9939(98)04468-2

\title{
MODULI OF PLANE CURVE SINGULARITIES WITH A SINGLE CHARACTERISTIC EXPONENT
}

\author{
ROSA PERAIRE
}

(Communicated by Ron Donagi)

\begin{abstract}
This paper studies the moduli space corresponding to irreducible germs of plane analytic curve with a single characteristic exponent. We stratify the moduli space corresponding to such germs using an analytical invariant introduced by Zariski. Then, we compute the minimum Tjurina number on each stratum as well as the dimension of the strata.
\end{abstract}

\section{INTRODUCTION}

Let $\gamma$ be an irreducible germ of plane analytic curve with a single characteristic exponent $\{m / n\}(n<m, \operatorname{gcd}(n, m)=1)$.

We say that an irreducible germ $\psi$ of plane analytic curve is $(n, m)$-quasihomogeneous if there are analytic coordinates where $\psi$ admits the equation: $y^{n}-x^{m}=0$.

Assume that $\gamma$ is non-quasihomogeneous. Then in [9], it is shown that there exists a parametric representation of $\gamma$ of the form

$$
\left\{\begin{array}{l}
x=t^{n} \\
y=t^{m}+b t^{m+s}+\sum_{i>s} b_{m+i} t^{m+i}, \quad b \in \mathbb{C}^{*},
\end{array}\right.
$$

in such a way that neither $m+s$ nor $n+s$ belongs to the semigroup generated by $n, m$. Moreover, the integer $s$ is the same for all such parametric representations of $\gamma$ and is therefore an analytical invariant. From now on we will call it $s(\gamma)$. In case $\gamma$ is quasihomogeneous we define $s(\gamma)=\infty$.

In this paper we study the stratification induced by the analytical invariant $s$ in the moduli space corresponding to irreducible germs of plane curve singularity with a single characteristic exponent.

In section 1, using the geometric interpretation of $s(\gamma)$ given by Casas [6], we give a universal family for all germs of plane curve singularity with fixed single characteristic exponent and fixed invariant $s$.

If $f=0$ is an equation of $\gamma$, we denote by $\tau(\gamma)$ the Tjurina number of $\gamma$, that is, $\tau(\gamma)=\operatorname{dim}_{\mathbb{C}} \mathbb{C}\{x, y\} /\left(f, \frac{\partial f}{\partial y}, \frac{\partial f}{\partial x}\right)$. Let us call $\tau_{\min }(n, m, s)$ the minimum of the integers $\tau(\gamma)$ when $\gamma$ ranges over the set of germs with characteristic exponent $\{m / n\}$ and fixed invariant $s$. In sections 2 and 3 , we adapt the algorithm of [3] to compute $\tau_{\min }(n, m, s)$.

Received by the editors April 19, 1996.

1991 Mathematics Subject Classification. Primary 14H20, 32S10.

Key words and phrases. Moduli, Tjurina number, equisingular miniversal deformation.

Supported by CAICYT PB91-0210-C02-02.

(C)1998 American Mathematical Society 
In section 4 , we compute the dimension of the $s$-constant strata in the moduli space corresponding to plane curve singularities with a single characteristic exponent.

I would like to thank E. Casas for useful discussions and suggestions.

\section{THE ANALYTICAL INVARIANT $s$}

(1.1) General conventions. Let $\gamma$ be an irreducible germ of plane analytic curve at the origin $O$ with a single characteristic exponent $\{m / n\}$. From now on we will assume that $\operatorname{gcd}(n, m)=1$ and $n<m$. Then, the semigroup of $\gamma,\langle n, m\rangle$, is $\langle n, m\rangle=n \mathbb{N}+m \mathbb{N}$, where we denote by $\mathbb{N}$ the set of non-negative integers.

We will denote by $[\xi \cdot \zeta]$ the intersection number of the germs $\xi$ and $\zeta$ at $O$.

(1.2). Let $\gamma$ be a germ of analytic curve with a single characteristic exponent $\{m / n\}$. Assume that local coordinates $\{x, y\}$ have been chosen so that the Puiseux expansion of $\gamma$ has the form

$$
\sigma(x)=x^{\frac{m}{n}}+\sum_{j \geq m+s} b_{j} x^{\frac{j}{n}}, \quad b_{m+s} \in \mathbb{C}^{*},
$$

with $n+s \notin\langle n, m\rangle, m+s \notin\langle n, m\rangle$. That is, $s=s(\gamma)$. Thus, an equation of $\gamma$ is

$$
f(x, y)=\prod_{\varepsilon^{n}=1}\left(y-\varepsilon^{m} x^{\frac{m}{n}}-\sum_{j \geq m+s} b_{j} \varepsilon^{j} x^{\frac{j}{n}}\right)=0
$$

and, by an easy computation, $f(x, y)$ may be written as

$$
f(x, y)=y^{n}-x^{m}+\sum_{n i+m j \geq m n+s} a_{i j} x^{i} y^{j}
$$

with $\sum_{n i+m j=s} a_{i j} \neq 0$. Conversely, if a germ is defined by the equation $f=0, f$ as above, then the Puiseux expansion of $\gamma$ has the form of (1.2.1).

(1.3). Let us recall that, by [6], theorem 3, one may also compute the analytical invariant $s(\gamma)$ as

$$
m n+s(\gamma)=\max \{[\psi \cdot \gamma] \mid \psi \text { an }(n, m) \text {-quasihomogeneous curve }\}
$$

Given two positive integers $n, m$ with $\operatorname{gcd}(n, m)=1$, we ask about all integers $s, s>0$ such that $s+m \notin\langle n, m\rangle$ and $s+n \notin\langle n, m\rangle$.

(1.4) Lemma. Let $A$ be the set

$$
A=\{s \in \mathbb{N} \mid s>0, s+m \notin\langle n, m\rangle, s+n \notin\langle n, m\rangle\},
$$

and let $B$ be the finite set

$$
B=\left\{(i, j) \in \mathbb{N}^{2} \mid n i+m j>m n, 0 \leq i \leq m-2,0 \leq j \leq n-2\right\} .
$$

Then, there is a bijection between $A$ and $B$ given by

$$
\begin{aligned}
B & \longrightarrow A \\
(i, j) & \longmapsto n i+m j-m n .
\end{aligned}
$$


Proof. Given $(i, j) \in B$, let $s=n i+m j-m n$. We will show that $s \in A$; that is, $s+m \notin\langle n, m\rangle$ and $s+n \notin\langle n, m\rangle$. Assume that $s+n \in\langle n, m\rangle$; then there exists $\left(i_{0}, j_{0}\right) \in \mathbb{N}^{2}$ such that $s+n=i_{0} n+j_{0} m$.

Since $(i, j) \in B$, then $0 \leq i \leq m-2,0 \leq j \leq n-2$, and hence $s+n<m n$. Now we claim that $j_{0}<n$; otherwise since $i_{0} \geq 0$, then $s+n=i_{0} n+j_{0} m \geq m n$, a contradiction. Thus, $j_{0}<n$, as claimed. On the other hand we may write

$$
m n+s=n i+m j=\left(i_{0}-1+m\right) n+j_{0} m .
$$

Thus,

$$
i=i_{0}-1+m+t m, \quad j=j_{0}-t n,
$$

for certain $t \in \mathbb{Z}$. As $0 \leq j \leq n-2$ and we have just proved that $0 \leq j_{0}<n$, necessarily $t=0$, so one gets $i=i_{0}-1+m \geq m-1$, and this is a contradiction because $i \leq m-2$. So $s+n \notin\langle n, m\rangle$, as wanted. In the same way one also proves that $s+m \notin\langle n, m\rangle$. Hence, $s \in A$ as claimed.

Next we will show the converse. Let $s \in A$. Thus, $s>0$. Since $\operatorname{gcd}(n, m)=1$, then the conductor of the semigroup $\langle n, m\rangle$ is $(n-1)(m-1)$. Therefore, $s+m n \in$ $\langle n, m\rangle$. So, there are $\left(i_{0}, j_{0}\right) \in \mathbb{N}^{2}$ such that $n i_{0}+m j_{0}=m n+s$. We claim that $\left(i_{0}, j_{0}\right) \in B$. We will prove that $0 \leq i_{0} \leq m-2$ and $0 \leq j_{0} \leq n-2$.

Since $(n-1)(m-1)$ is the conductor of the semigroup $\langle n, m\rangle$, and $s+m \notin\langle n, m\rangle$, $s+n \notin\langle n, m\rangle$, then $\max (s+m, s+n)<(m-1)(n-1)$. Assume now that $i_{0}>m-2$; this would imply that

$$
s+n=n i_{0}+m j_{0}-m n+n=n\left(i_{0}-m+1\right)+m j_{0} \in\langle n, m\rangle,
$$

a contradiction. Hence, $i_{0} \leq m-2$. In the same way one shows that $j_{0} \leq n-2$, so this concludes the proof.

(1.5) Theorem. Let $\gamma$ be an irreducible germ of analytic curve at $O$ with characteristic exponent $\{m / n\}$. Let $s$ be

$$
s=\max \{[\psi \cdot \gamma] \mid \psi \text { an }(n, m) \text {-quasihomogeneous curve }\}-m n .
$$

Then:

a) $s>0$, and $s=\infty$ if and only if $\gamma$ is $(n, m)$-quasihomogeneous.

b) If $s$ is finite, then there are analytic coordinates $\{x, y\}$ such that $\gamma$ has equation

$$
y^{n}-x^{m}+x^{p} y^{q}+\sum_{\substack{n i+m j>m n+s \\ 0 \leq i \leq m-2 \\ 0 \leq j \leq n-2}} a_{i j} x^{i} y^{j}=0,
$$

for certain $(p, q) \in B$ (B as in lemma (1.4)) with $n p+m q=m n+s$.

Proof. Claim a) follows directly from (1.3). Let us show part b). By [10], VI.2.1, using suitable coordinates $\{x, y\}$ one may assume that $\gamma$ has equation

$$
y^{n}-x^{m}+\sum_{(i, j) \in B} a_{i j} x^{i} y^{j}=0 .
$$

Let $k=\min \left\{n i+m j \mid a_{i j} \neq 0\right\}$. By (1.3) and (1.4), $s \geq k-m n$. On the other hand, by (1.4), $k-m n+m \notin\langle n, m\rangle, k-m n+n \notin\langle n, m\rangle$; then, by (1.2), $s=k-m n$. 
Let $(p, q)$ be the only element in $B$ such that $n i+m j=m n+s$. Necessarily (1.5.1) has the form

$$
y^{n}-x^{m}+a_{p q} x^{p} y^{q}+\sum_{\substack{n i+m j>m n+s \\ 0 \leq i \leq m-2 \\ 0 \leq j \leq n-2}} a_{i j} x^{i} y^{j}=0, \quad a_{p q} \in \mathbb{C}^{*} .
$$

Let $\alpha \in \mathbb{C}^{*}$ be such that $\alpha^{s}=a_{p q}$. Then, in coordinates $\{\bar{x}, \bar{y}\}, \bar{x}=\alpha^{n} x, \bar{y}=\alpha^{m} y$, $\gamma$ has equation

$$
\bar{y}^{n}-\bar{x}^{m}+\bar{x}^{p} \bar{y}^{q}+\sum_{\substack{n i+m j>m n+s \\ 0 \leq i \leq m-2 \\ 0 \leq j \leq n-2}} \bar{a}_{i j} \bar{x}^{i} \bar{y}^{j}=0, \quad \bar{a}_{i j}=a_{i j} / \alpha^{n i+m j-m n},
$$

as claimed.

\section{Description of the ALGORIthm}

By (1.5), all irreducible germs of plane analytic curve with characteristic exponent $\{m / n\}$ and fixed invariant $s$ are analytically equivalent to a germ defined by an equation belonging to the family $F(x, y, \mathbf{a})=0$, where

$$
F(x, y, \mathbf{a})=y^{n}-x^{m}+x^{p} y^{q}+\sum_{\substack{n i+m j>m n+s \\ 0 \leq i \leq m-2 \\ 0 \leq j \leq n-2}} a_{i j} x^{i} y^{j}
$$

and $n p+m q=m n+s, 0 \leq p \leq m-2,0 \leq q \leq m-2$. Call $N_{s}=\#\left(\left\{(i, j) \in \mathbb{N}^{2} \mid\right.\right.$ $n i+m j>m n+s, 0 \leq i \leq m-2,0 \leq j \leq n-2\})$.

In this section we give an algorithm to compute the Tjurina number for any germ with equation $F(x, y, \mathbf{a})=0, F$ as above, when the coefficients $\mathbf{a}=\left(a_{i j}\right)$ belong to a certain non-empty Zariski open set of $\mathbb{C}^{N_{s}}$. From now on we will call such germs generic.

First we will introduce some notations.

(2.1). From now on we fix the positive linear form $\rho(i, j)=n i+m j$. Let $S \subset \mathbb{N}^{2}$ be a non-empty subset; we define $\rho(S)=\min \{\rho(i, j) \mid(i, j) \in S\}$ and $\exp (S)=(\alpha, \beta)$ if $(\alpha, \beta) \in S, \rho(\alpha, \beta)=\rho(S)$ and $\alpha=\min \{i \mid(i, j) \in S, \rho(i, j)=\rho(S)\}$. Consider in $\mathbb{N}^{2}$ the total ordering induced by $\rho$ :

$$
(i, j)<\left(i^{\prime}, j^{\prime}\right) \Longleftrightarrow \begin{cases}\rho(i, j)<\rho\left(i^{\prime}, j^{\prime}\right), & \text { or } \\ \rho(i, j)=\rho\left(i^{\prime}, j^{\prime}\right) & \text { and } i<i^{\prime} .\end{cases}
$$

Note that $\exp (S)$ is the minimum of $S$ with respect to this ordering.

(2.2) Description of the algorithm. First we define

$$
\Lambda_{-1}=(0, n-1), \quad \Lambda_{0}=(m-1,0), \quad \Lambda_{1}=(p, q),
$$

where $n p+m q=m n+s, 0 \leq p \leq m-2,0 \leq q \leq m-2$. We define $\Omega_{-1}$ as

$$
\Omega_{-1}=\mathbb{N}^{2} \backslash\left(\Lambda_{-1}+\mathbb{N}^{2}\right) \text {. }
$$

Assume that we have defined $\Lambda_{r}$, for $r=-1, \ldots, \ell$. From them, we define the subsets $\Omega_{r}$ of $\mathbb{N}^{2}$ as

$$
\Omega_{r}=\Omega_{r-1} \backslash\left(\Lambda_{r}+\mathbb{N}^{2}\right), \quad r=0, \ldots, \ell,
$$


and let $W_{r}$ be the minimal set in $\mathbb{N}^{2}$ such that

$$
\bigcup_{j=1}^{r}\left(\Lambda_{j}+\mathbb{N}^{2}\right)=W_{r}+\left(\mathbb{N}^{2} \backslash(\mathbb{N} \times\{0\} \cup\{0\} \times \mathbb{N})\right)
$$

(see Figure (2.2.1)).

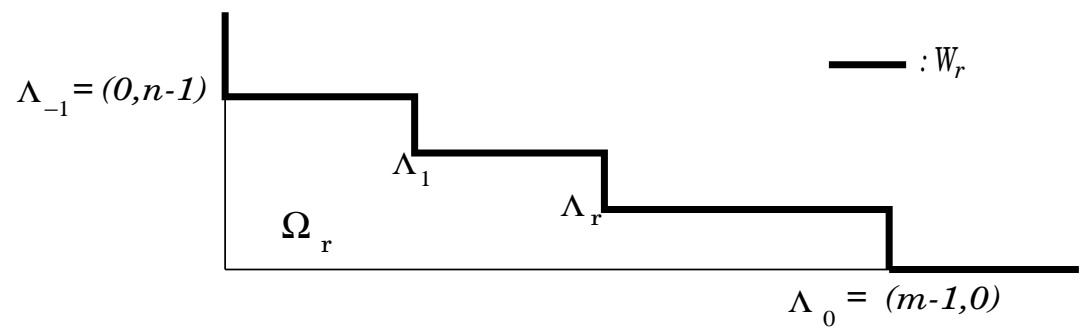

Figure (2.2.1)

(2.3) Step $\ell+1$. We will now construct $\Lambda_{\ell+1}$. Write $\Lambda_{\ell}=\left(a_{\ell}, b_{\ell}\right)$ and let $U_{\ell}=$ $\left(u_{\ell}, b_{\ell}\right) \in W_{\ell}, V_{\ell}=\left(a_{\ell}, v_{\ell}\right) \in W_{\ell}$ be such that $\left(u_{\ell}+1, b_{\ell}\right) \notin W_{\ell}$ and $\left(a_{\ell}, v_{\ell}+1\right) \notin W_{\ell}$ (see Figure (2.3.1)). Then, define

$$
\begin{aligned}
d_{\ell} & =\min \left(\rho\left(U_{\ell}\right), \rho\left(V_{\ell}\right)\right), \\
S_{\ell+1} & =\left\{(i, j) \in \Omega_{\ell} \mid \rho(i, j)>d_{\ell}\right\} .
\end{aligned}
$$

If $S_{\ell+1}=\emptyset$, the algorithm finishes at step $\ell+1$. Otherwise, we define $\Lambda_{\ell+1}$ as

$$
\Lambda_{\ell+1}=\exp \left(S_{\ell+1}\right) \text {. }
$$

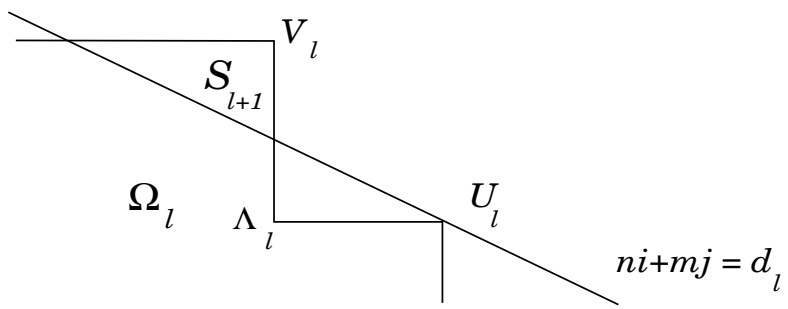

Figure (2.3.1)

(2.4) Lemma. The algorithm finishes in a finite number of steps.

Proof. On one hand, by the definition of the sets $\Omega_{\ell}$ we have $\Omega_{\ell-1} \supseteq \Omega_{\ell}$ for any $\ell$ th step in the algorithm. On the other hand, $\Omega_{0}=\left\{(i, j) \in \mathbb{N}^{2} \mid 0 \leq i \leq m-2,0 \leq\right.$ $j \leq n-2\}$ is a finite subset of $\mathbb{N}^{2}$, so the claim follows.

(2.5). Let us assume that the algorithm finishes at step $M+1$. From now on we will write $\Omega=\Omega_{M}$. 


\section{A STANDARD BASIS FOR THE JACOBIAN IDEAL OF A GENERIC GERM WITH CHARACTERISTIC EXPONENT $\{m / n\}$ AND FIXED $s$}

In this section we will use the notions of privileged exponents with respect to a direction $\rho\left(\exp _{\rho}\right)$, standard basis of an ideal, and the associated division theorem. For this concepts we refer the reader to [1] and [2].

(3.1). Consider

$$
F(x, y, \mathbf{a})=y^{n}-x^{m}+x^{p} y^{q}+\sum_{\substack{n i+m j>m n+s \\ 0 \leq i \leq m-2 \\ 0 \leq j \leq n-2}} a_{i j} x^{i} y^{j},
$$

where $n p+m q=m n+s>m n, 0 \leq p \leq m-2$ and $0 \leq q \leq n-2$.

We will compute a standard basis for the jacobian ideal, $\left(F, \frac{\partial F}{\partial x}, \frac{\partial F}{\partial y}\right)$, with respect to the direction $\rho(i, j)=n i+m j$ in the case when the coefficients $\mathbf{a}=\left(a_{i j}\right)$ are generic, that is, $\mathbf{a}=\left(a_{i j}\right)$ belong to a Zariski open set of $\mathbb{C}^{N_{s}}$.

We will use the following notation: for $I=(i, j)$ we denote by $z^{I}$ the monomial $x^{i} y^{j}$. In $\mathbb{C}\{x, y\}$, we fix the monomial ordering $<_{\rho}$ :

$$
x^{i} y^{j}<_{\rho} x^{i^{\prime}} y^{j^{\prime}} \Longleftrightarrow \begin{cases}\rho(i, j)<\rho\left(i^{\prime}, j^{\prime}\right) & \text { or } \\ \rho(i, j)=\rho\left(i^{\prime}, j^{\prime}\right) & \text { and } i<i^{\prime} .\end{cases}
$$

Then, $\exp _{\rho}\left(\sum g_{i j} x^{i} y^{j}\right)=(\alpha, \beta)$ means that $x^{\alpha} y^{\beta}$ is the minimum of the set $\left\{x^{i} y^{j} \mid\right.$ $\left.g_{i j} \neq 0\right\}$ with respect to this ordering.

The proofs of lemma (3.2) and proposition (3.4) are the same as the ones of III.1 in $[3]$.

(3.2) Lemma (cf. [3], III.1 lemma). There is a non-empty Zariski open set $\mathcal{Z}$ in the parameter space of the $\mathbf{a}=\left(a_{i j}\right)$ such that for $\mathbf{a} \in \mathcal{Z}$, there are $G_{\ell}=\sum g_{\ell, I}(\mathbf{a}) z^{I}$ $\in\left(F, \frac{\partial F}{\partial y}, \frac{\partial F}{\partial x}\right)$ with $\exp _{\rho}\left(G_{\ell}\right)=\Lambda_{\ell}$ for $\ell=1, \ldots, M$. Precisely, for any $\ell=$ $1, \ldots, M-1, G_{\ell+1}$ is the remainder on division of $z^{I_{\ell}} G_{\ell}$ by $G_{-1}, G_{0}, \ldots, G_{\ell}$.

(3.3). After suitable shrinking of $\mathcal{Z}$ one may assume all $g_{\ell, \Lambda_{\ell}}, \ell=1, \ldots, M$, to be nonzero in $\mathcal{Z}$. Then, we have,

(3.4) Proposition (cf. [3], III.1 proposition). There exists a Zariski non-empty open set $\mathcal{Z}$ in the parameter space such that for every $\mathbf{a} \in \mathcal{Z}$ the escalier of the ideal $\left(F, \frac{\partial F}{\partial y}, \frac{\partial F}{\partial x}\right)$ is $\left\{\Lambda_{\ell}\right\}_{\ell=-1}^{M}$.

(3.5) Theorem. Let $\gamma$ be an irreducible germ of curve at $O$ with characteristic exponent $\{m / n\}$, fixed finite invariant $s$ and defined by the equation

$$
y^{n}-x^{m}+x^{p} y^{q}+\sum_{\substack{n i+m j>s \\ 0 \leq i \leq m-2 \\ 0 \leq j \leq n-2}} a_{i j} x^{i} y^{j}=0,
$$

where $n p+m q=m n+s, 0 \leq p \leq m-2$ and $0 \leq j \leq n-2$. Assume that the coefficients $\left(a_{i j}\right)$ belong to the Zariski open set $\mathcal{Z}$ (see (3.3) for the definition). Then, $\tau(\gamma)=\# \Omega$, where $\Omega$ is given by the algorithm of section 2 (see (2.5)). 
Proof. In (3.2) and (3.4), we have computed a standard basis and the escalier with respect to the direction $\rho(i, j)=n i+m j$ for the jacobian ideal of $\gamma$ in the case $\left(a_{i j}\right) \in \mathcal{Z}$. Therefore, by [2], I.1.12, the claim follows.

Since $\tau(\gamma)$ is upper semicontinuous, $\tau_{\min }(n, m, s)$ is given by $\tau(\gamma)$ for a generic $\gamma$. So, theorem (3.5) leads to

(3.6) Corollary. $\tau_{\min }(n, m, s)=\#(\Omega)$, where $\Omega$ is given by the algorithm of section 2 .

(3.7) Remark. By using Buchberger's algorithm [4] for the computation of a standard basis, one easily obtains a standard basis for the jacobian ideal of the germ $\gamma_{p q}: y^{n}-x^{m}+x^{p} y^{q}=0(n p+m q>m n, 0 \leq p \leq m-2,0 \leq q \leq n-2)$. In general, $\tau\left(\gamma_{p q}\right)$ does not give the maximum of the integers $\tau(\gamma)$ when $\gamma$ ranges over the set of germs with characteristic exponent $\{m / n\}$ and fixed invariant $s=n p+m q-m n$. For instance, for the family corresponding to the characteristic exponent $\{27 / 8\}$ and $s=17$, the germ defined by $y^{8}-x^{27}+x^{19} y^{3}=0$ has Tjurina number 153 while the germ defined by

$$
y^{8}-x^{27}+x^{19} y^{3}-\frac{19}{54} x^{11} y^{6}=0
$$

has Tjurina number 154 (computations made by using CoCoA [5]).

\section{Moduli for Plane Curve Singularities With a SINGLE CHARACTERISTIC EXPONENT $\{m / n\}$ AND FIXED INVARIANT $s$.}

In this section we stratify the moduli space corresponding to plane curve singularities with a single characteristic exponent using the analytical invariant $s$, and we compute the dimension of an open dense subset of each stratum.

(4.1). Let $N$ be

$$
N=\#\left\{(i, j) \in \mathbb{N}^{2} \mid n i+m j>m n, 0 \leq i \leq m-2,0 \leq j \leq n-2\right\} .
$$

By [8], I.2.10, $\mathbb{C}^{N}$ is the base space of a representative $(X, 0) \underset{\Sigma}{\stackrel{H}{\rightleftarrows}}\left(\mathbb{C}^{N}, 0\right)$ of the equisingular miniversal deformation of the germ at the origin of the $(n, m)$-quasihomogeneous curve: $y^{n}-x^{m}=0$, where $\Sigma$ is the section which picks up the only singular point in each fibre (i.e., the origin). Analytical equivalence of germs induces an equivalence relation $\sim$ in $\mathbb{C}^{N}$. The moduli space corresponding to the characteristic exponent $\{m / n\}$ will be denoted $\mathcal{M}_{(n, m)}$; it is defined (see [8], II.2.2) as the topological space $\mathbb{C}^{N} / \sim$ (with the quotient topology). Let $\pi: \mathbb{C}^{N} \longrightarrow \mathcal{M}_{(n, m)}$ be the canonical surjective map.

(4.2). For any $s \in \mathbb{N}, s+m \notin\langle n, m\rangle, s+n \notin\langle n, m\rangle$, we have defined in section 2 the following integer:

$$
N_{s}=\#\left\{(i, j) \in \mathbb{N}^{2} \mid n i+m j>m n+s, 0 \leq i \leq m-2,0 \leq j \leq n-2\right\} .
$$

Let $(p, q)$ be the only element in $B(B$ as in (1.4)) satisfying $n p+m q=m n+s$. In $\mathbb{C}^{N_{s}+1}$ we consider the open set

$$
\mathcal{U}_{p q}=\left\{\left(a_{p q},\left(a_{i j}\right)\right) \in \mathbb{C} \times \mathbb{C}^{N_{s}} \mid a_{p q} \neq 0\right\} .
$$


Let $H_{s}: \mathbb{C}^{2} \times\left(\mathcal{U}_{p q} \cup\{0\}\right) \longrightarrow\left(\mathcal{U}_{p q} \cup\{0\}\right)$ be the deformation of the germ at the origin: $y^{n}-x^{m}=0$, whose fibre at the point $\left(a_{p q},\left(a_{i j}\right)\right) \in \mathcal{U}_{p q} \cup\{0\}$ is the germ defined by the equation

$$
y^{n}-x^{m}+a_{p q} x^{p} y^{q}+\sum_{\substack{n i+m j>m n+s \\ 0 \leq i \leq m-2 \\ 0 \leq j \leq n-2}} a_{i j} x^{i} y^{j}=0 .
$$

(4.3) Lemma. All fibres of the deformation $H_{s}: \mathbb{C}^{2} \times\left(\mathcal{U}_{p q} \cup\{0\}\right) \longrightarrow\left(\mathcal{U}_{p q} \cup\{0\}\right)$ are equisingular and all fibres but the one at the origin have constant invariant $s$. Moreover, $H_{s}: \mathbb{C}^{2} \times\left(\mathcal{U}_{p q} \cup\{0\}\right) \longrightarrow\left(\mathcal{U}_{p q} \cup\{0\}\right)$ is miniversal for such deformations.

Proof. The proof is analogous to that of [8], I.2.10, but in this case may be shortened by using (1.5).

As in (4.1), analytical equivalence of germs induces an equivalence relation $\sim$ in $\mathcal{U}_{p q}$.

(4.4) Definition. We will call the moduli space corresponding to the characteristic exponent $\{m / n\}$ and to the invariant $s$, which will be denoted by $\mathcal{M}_{(n, m, s)}$, the topological space $\mathcal{U}_{p q} / \sim$ (with the quotient topology). Let $\pi_{s}: \mathcal{U}_{p q} \longrightarrow \mathcal{M}_{(n, m, s)}$ be the canonical surjective map.

(4.5) Proposition. $\mathcal{M}_{(n, m, s)}$ is connected and locally closed in $\mathcal{M}_{(n, m)}$.

Proof. The connectedness follows directly from (1.5). To show that $\mathcal{M}_{(n, m, s)}$ is locally closed, we again use (1.5). Namely,

$$
\begin{array}{r}
\pi^{-1}\left(\mathcal{M}_{(n, m, s)}\right)=\left\{\left(a_{i j}\right) \in \mathbb{C}^{N} \mid a_{i j}=0 \text { if } n i+m j<m n+s\right\} \\
\cap\left\{\left(a_{i j}\right) \in \mathbb{C}^{N} \mid a_{i j} \neq 0 \text { if } n i+m j=m n+s\right\}
\end{array}
$$

which is clearly locally closed in $\mathbb{C}^{N}$, and so, the claim follows.

(4.6). For $\mathbf{b}=\left(b_{p q},\left(b_{i j}\right)\right) \in \mathcal{U}_{p q} \subset \mathbb{C}^{N_{s}+1}\left(\mathcal{U}_{p q}\right.$ as in (4.2)) we define

$$
G(x, y, \mathbf{b})=y^{n}-x^{m}+b_{p q} x^{p} y^{q}+\sum_{\substack{n i+m j>m n+s \\ 0 \leq i \leq m-2 \\ 0 \leq j \leq n-2}} b_{i j} x^{i} y^{j} .
$$

So, $G\left(x, y,\left(1,\left(b_{i j}\right)\right)\right)=F\left(x, y,\left(b_{i j}\right)\right)(F$ as in (3.1)). The analytical change of coordinates $x=u^{n} \bar{x}, y=u^{m} \bar{y}, u \in \mathbb{C}^{*}$, transforms the germ at the origin defined by the equation $G(x, y, \mathbf{b})=0$ into the germ defined by the equation

$$
G\left(\bar{x}, \bar{y}, u^{s} b_{p q},\left(u^{n i+m j-m n} b_{i j}\right)\right)=0 .
$$

Thus, for any $\varepsilon>0, B_{\varepsilon}(0) \cap \mathcal{U}_{p q}$ maps onto the moduli space $\mathcal{M}_{(n, m, s)}$.

Let us denote by $\tau(\mathbf{b})$ the Tjurina number of the germ at the origin defined by $G(x, y, \mathbf{b})=0$. Consider the following subset of $\mathcal{M}(n, m, s)$ :

$$
\mathcal{M}_{(n, m, s)}\left(\tau_{\min }\right)=\pi_{s}\left(\left\{\mathbf{b} \in \mathcal{U}_{p q} \mid \tau(\mathbf{b})=\tau_{\min }(n, m, s)\right\}\right) .
$$

(4.7) Proposition. $\mathcal{M}_{(n, m, s)}\left(\tau_{\min }\right)$ is an open dense subset of $\mathcal{M}_{(n, m, s)}$.

Proof. Since the Tjurina number is upper semicontinuous, $\mathcal{M}_{(n, m, s)}\left(\tau_{\min }\right)$ is open in $\mathcal{M}_{(n, m, s)}$, and from (3.5) it follows that it is dense. 
(4.8). Given $\mathbf{b} \in \mathcal{U}_{p q}$, denote by $(X(\mathbf{b}), 0) \longrightarrow\left(S_{(n, m, s)}(\mathbf{b}), 0\right)$ the equisingular miniversal deformation with fixed invariant $s$ of the germ at the origin defined by $G(x, y, \mathbf{b})=0$. We consider the following open subset of $B_{\varepsilon}(0) \cap \mathcal{U}_{p q}$ :

$$
V_{p q}=\left\{\mathbf{b} \in B_{\varepsilon}(0) \cap \mathcal{U}_{p q} \mid \tau(\mathbf{b})=\tau_{\min }(n, m, s)\right\} ;
$$

by (3.5) it is dense in $B_{\varepsilon}(0) \cap \mathcal{U}_{p q}$.

(4.9) Lemma. If $\mathbf{b} \in V_{p q}$, then there exists a neighbourhood $V_{\mathbf{b}}$ of $\mathbf{b}$ in $S_{(n, m, s)}(\mathbf{b})$ such that $V_{\mathbf{b}}$ maps one-to-one in the moduli space $\mathcal{M}_{(n, m, s)}$.

Proof. Since $\tau(\mathbf{b})=\tau_{\min }(n, m, s)$ and the Tjurina number is upper semicontinuous, in a neighbourhood of $\mathbf{b}$ in $S_{(n, m, s)}(\mathbf{b})$ the Tjurina number must be constant (and equal to $\left.\tau_{\min }(n, m, s)\right)$. Then, the claim follows directly from [8], III.2.9.2.

The above lemma gives

(4.10) Corollary. $\operatorname{dim} \mathcal{M}_{(n, m, s)}\left(\tau_{\min }\right)=\operatorname{dim} S_{(n, m, s)}(\mathbf{b})$ for $\mathbf{b} \in V_{p q}$.

Let us now compute the dimension of $S_{(n, m, s)}(\mathbf{b})$.

(4.11) Proposition. If $\mathbf{b} \in \mathcal{U}_{p q} \cap B_{\varepsilon}(0)$ (for a small enough $\varepsilon>0$ ), then

$$
\operatorname{dim} S_{(n, m, s)}(\mathbf{b})=N_{s}+1-\mu+\tau(\mathbf{b}),
$$

where $\mu$ is the Milnor number of the equisingularity class corresponding to the characteristic exponent $\{m / n\}$ (i.e., $\mu=(n-1)(m-1))$.

Proof. By [8], III.2.1, for a small enough $\varepsilon>0$, the germ of $\mathcal{U}_{p q} \subset \mathbb{C}^{N_{s}+1}$ at the point $\mathbf{b} \in \mathcal{U}_{p q} \cap B_{\varepsilon}(0)$ may be written as

$$
\left(\mathcal{U}_{p q}, \mathbf{b}\right) \simeq\left(\mathbb{C}^{\mu-\tau(\mathbf{b})} \times S_{(n, m, s)}, 0\right) .
$$

So, $\operatorname{dim} S_{(n, m, s)}(\mathbf{b})=N_{s}+1-\mu+\tau(\mathbf{b})$.

From (4.10) and (4.11) one concludes that

(4.12) Theorem. $\operatorname{dim} \mathcal{M}_{(n, m, s)}\left(\tau_{\min }\right)=N_{s}+1-\mu+\tau_{\min }(n, m, s)$.

(4.13) Remark. Laudal and Pfister in [7] stratify the moduli space corresponding to a single characteristic exponent by using the Tjurina number. Their stratification is not comparable to ours. For instance, for the equisingularity class corresponding to the characteristic exponent $\{11 / 5\}$ the germs defined by the equations

$$
\begin{aligned}
& y^{5}-x^{11}+x^{9} y+x^{7} y^{2}+x^{6} y^{3}=0, \\
& y^{5}-x^{11}+x^{7} y^{2}+x^{6} y^{3}=0
\end{aligned}
$$

have Tjurina number 34 and invariant $s=1, s=2$ respectively. On the other hand, the germs

$$
\begin{aligned}
& y^{5}-x^{11}+x^{9} y=0, \\
& y^{5}-x^{11}+x^{9} y+x^{7} y^{2}+x^{6} y^{3}=0
\end{aligned}
$$

both have invariant $s=1$ and Tjurina number 35, 34 respectively. (Computations made by using $\mathrm{CoCoA}$ [5].) 


\section{REFERENCES}

[1] Briançon, J., Weierstrass préparé à la Hironaka, Astérisque $\mathbf{7}$ et 8 (1973), 67-73. MR 50:13584

[2] Briançon, J., Description de Hilb $\mathbb{C}^{n}\{x, y\}$, Invent. Math. 41 (1977), 45-89. MR 56:15637

[3] Briançon, J., Granger, M., Maisonobe, Ph., Le nombre de modules du germe de courbe plane $x^{a}+y^{b}=0$, Math. Ann. 279 (1988), 535-551. MR 89c: 14001

[4] Buchberger, B., Ein algorithmus zum auffiden der basiselemente des restklassenringes nach einem nulldimensionalen polynomideal, Thesis Univ. Insbruck, 1965.

[5] Capani, L., Niesi, A., Robbiano, L., CoCoA: A system for doing computations in commutative algebra, Department of Mathematics, University of Genova. e-mail: cocoa@ dima.unige.it.

[6] Casas-Alvero, E., Moduli of algebroid plane curves, Algebraic Geometry, La Rábida 1981, Springer-Verlag. Lecture Notes in Math, 961, Berlin, 1982, pp. 32-83. MR 85g:14028

[7] Laudal, O.A., Pfister, G., Local moduli and singularities, Springer-Verlag, Lecture Notes in Math. 1310, 1988. MR 89h:14008

[8] Teissier, B., Appendix to [10]. MR 54:2662

[9] Zariski, O., Characterization of plane algebroid curves whose module of differentials has maximum torsion, Proceedings Nat. Acad. of Science U.S.A 56 (1966), 781-786. MR 34:2576

[10] Zariski, O., Le problème des modules pour les branches planes, Centre de Math., Ecole Polytechnique, Paris, 1973. MR 54:2662

Departament d'Algebra i Geometria, Universitat de Barcelona. Gran Via, 585, 08007 BARCELONA, Spain

E-mail address: peraire@cerber.mat.ub.es 